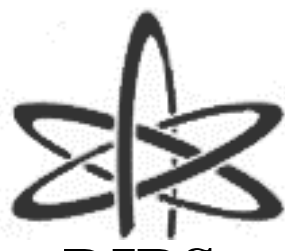

BJRS
BRAZILIAN JOURNAL

$\mathrm{OF}$

RADIATION SCIENCES

03-01 (2015) 01-15

\title{
Enfermagem radiológica e os riscos ocupacionais: Estado da arte
}

\author{
J. A. C. Melo ${ }^{\mathrm{a}, \mathrm{b}}$, K. R. Luz ${ }^{\mathrm{b}}$, A. Huhn', E. Viana ${ }^{\mathrm{a}}$, G. Rosa ${ }^{\mathrm{a}}$, R. Derech ${ }^{\mathrm{c}}$ \\ ${ }^{a}$ Instituto Federal de Educação, Ciência e Tecnologia de Santa Catarina - IFSC. \\ ${ }^{b}$ Universidade Federal de Santa Catarina - UFSC \\ ${ }^{c}$ Prefeitura Municipal de Florianópolis \\ julianac@ifsc.edu.br
}

\begin{abstract}
Documentary research with the aim to reflect on the state of the art in radiological nursing, on the nursing work and occupational hazards they are exposed, from articles produced in Brazil. The survey was conducted in the BVS, the study consisted of seven articles published from 2002 to 2012. Regarding the main variables studied in the articles, issues emerged related to working conditions and biosafety, performance of nurses, legislation, continuing education and difficulties and needs of nursing staff . It was noticed that is recent the interest in the field, but the work of nurses in radiological technologies must be grounded in current scientific knowledge and with proper technique, to the achievement of the desired clinical outcome and to promote their safety, the team and also the patient.
\end{abstract}

Keywords: Radiation Protection. Technology Radiologic. Occupational Health Nursing. 


\section{INTRODUCTION}

O processo de trabalho na área da saúde é realizado por diversas categorias profissionais, dentro das quais se encontra a enfermagem ${ }^{(1)}$. A Enfermagem é uma profissão que possui significativo contingente de profissionais atuando em diversos lugares e desenvolvendo as mais variadas funções dentro da área da saúde ${ }^{(2)}$.

A atuação dos enfermeiros na área de radiodiagnóstico partiu principalmente da necessidade de cumprir o que determina a resolução do Conselho Federal de Enfermagem (COFEN) $n^{\circ} 347 / 2009^{(3)}$ esta resolução determina que sempre que houver ações de enfermagem sendo executadas, é indispensável a presença e responsabilidade de um enfermeiro no local.

A competência do Enfermeiro em radioterapia, medicina nuclear e serviços por imagem estão estabelecido na Resolução do COFEN n ${ }^{\circ}$ 211/98 ${ }^{(4)}$ destacando entre outras funções a de planejar, organizar, supervisionar, executar e avaliar todas as atividades de Enfermagem, baseados em uma metodologia assistencial de Enfermagem; participar de programas de garantia da qualidade em serviços que utilizam radiação ionizante, de forma setorizada e global; proporcionar condições para o aprimoramento dos profissionais de Enfermagem atuantes na área, através de cursos e estágios em instituições afins; registra informações e dados estatísticos pertinentes à assistência de Enfermagem, ressaltando os indicadores de desempenho, interpretando e otimizando a utilização dos mesmos e manter atualização técnica e científica de manuseio dos equipamentos de radioproteção, que lhe permita atuar com eficácia em situações de rotina e emergenciais, visando interromper e/ou evitar acidentes ou ocorrências que possam causar algum dano físico ou material.

A enfermagem radiológica é especialidade da enfermagem relacionada ao cuidado do usurário ou ocorrências que possam causar algum dano físico ou material.

A enfermagem radiológica é a especialidade da enfermagem relacionada ao cuidado do usuário submetido a procedimentos diagnósticos e terapêuticos nos Serviços de Radiologia e Diagnóstico por Imagem - (OPAS) ${ }^{(7)}$. 
Esta especialidade vem sofrendo grandes modificações desde a descoberta dos raios $\mathrm{X}$ por Roentgen, em 1895. A radiação X tem sido aplicada em grande escala na área da saúde para fins terapêuticos e diagnóstico, contribuindo para o desenvolvimento tecnológico da área da saúde e qualidade de vida dos pacientes e usuários. Devido a estas transformações e o crescimento da atuação da enfermagem ao longo dos anos é necessário o aperfeiçoamento técnico - científico, sendo imprescindível a qualificação da enfermagem para atuar com conhecimento, segurança e qualidade em seu processo de trabalho nessa especialidade ${ }^{(6)}$.

Em decorrência de uma demanda de atendimentos a pacientes com necessidade cada vez mais complexas, é exigido do setor de saúde a incorporação de novas tecnologias e formas de organização do trabalho. Porém, esta nova configuração pode contribuir para o adoecimento dos profissionais decorrente da exposição insegura a riscos ocupacionais ${ }^{(2)}$.

Os fatores de riscos ocupacionais, de acordo com a Organização Pan-Americana de Saúde $(\mathrm{OPAS})^{(7)}$,são classificados em químicos, biológicos, ergonômicos, psicossociais, mecânicos, de acidentes e físicos. Os agentes físicos englobam diversas formas de energia a que possam estar expostos os profissionais, tais como vibração, pressão, ruído, temperaturas extremas, radiações não ionizantes e radiações ionizantes.

Procurando entender e visualizar tal processo de produção de conhecimento este artigo tem por objetivo refletir sobre o estado da arte na enfermagem radiológica acerca da atuação e os riscos ocupacionais que estão expostos, a partir de artigos produzidos no Brasil.

A credita-se que o resultado deste estudo contribuirá para dar visibilidade à produção científica sobre a temática da enfermagem radiológica, identificar as lacunas existentes, e assim poderá ser utilizado em planejamentos e estratégicas que direcionem pesquisas sobre os temas ainda pouco explorados nos Cursos de Pós-Graduação em Enfermagem. A divulgação da temática pesquisada poderá fornecer subsídios para que os docentes, discentes e pesquisadores das referidas áreas possa, direcionar as investigações sobre os aspectos relevantes nos diversos cenários do cuidado em relação a enfermagem radiológica. 


\section{MATERIAIS E MÉTODOS}

Trata-se de um estudo sobre o estado da arte realizado por meio de publicações científicas acerca da atuação do enfermeiro na enfermagem radiológica e os riscos ocupacionais a que estão expostos.

O estado da arte ou estado do conhecimento é uma metodologia utilizada com objetivo principal de organização teórica de um campo e/ou temática específica, eles parecem trazer em comum o desafio de mapear e de discutir uma produção acadêmica em diferentes campos do conhecimento, tentando responder de que formas e em que condições têm sido produzidas pesquisas de levantamento e avaliação do conhecimento sobre determinado tema, a partir da necessidade de conhecer o já construído e produzido para buscar o que ainda não foi feito ${ }^{(8)}$.

Inicialmente a busca se deu por consulta dirigida aos resumos dos artigos cujo título evidenciasse qualquer relação com o tema, foram usadas palavras-chave casadas para efetuar a busca, a tabela 1 demonstra os resulatdos:

Tabela 1: Relação das palavras - chaves e seus resultados

\begin{tabular}{|c|c|c|c|c|}
\hline Palavra Chave & $\begin{array}{l}\text { Total de } \\
\text { artigos }\end{array}$ & $\begin{array}{c}\text { Texto } \\
\text { complementos }\end{array}$ & $\begin{array}{c}\text { Escritos em } \\
\text { português }\end{array}$ & Aderência ao tema \\
\hline $\begin{array}{c}\text { Enfermagem e } \\
\text { Radiologia }\end{array}$ & 461 & 87 & 12 & 03 \\
\hline $\begin{array}{c}\text { Riscos } \\
\text { ocupacionais e } \\
\text { radiologia }\end{array}$ & 17 & 07 & 04 & 04 \\
\hline $\begin{array}{c}\text { Atuação da } \\
\text { enfermagem em } \\
\text { radiologia }\end{array}$ & 03 & 02 & 02 & 02 \\
\hline
\end{tabular}


O rastreamento realizado empregou estudos primários optando-se por acessar a Biblioteca Virtual em Saúde (BVS), disponível no endereço eletrônico http://www.bireme.br onde foram pesquisados vários bancos de dados de periódicos.

As seguintes etapas foram seguidas:

a) Definição de critérios de seleção da amostra definindo critérios de inclusão e exclusão dos artigos;

b) Definição das informações a serem extraídas dos artigos;

c) Análise dos resultados do conteúdo dos artigos;

d) Destaque aos artigos que fazem uma associação da atuação do enfermeiro e os riscos a enfermagem radiológica.

Entretanto, para o refinamento adequado da pesquisa, foi definido aos seguintes critérios de inclusão: artigos em português, com os resumos disponíveis, tratarem de enfermagem radiológica como principal ou como aspecto relevante na pesquisa e artigos publicados de 2002 a 2012. E artigos que utilizassem alguma critério metodológico. No entanto, é válido destacar que outros estudos podem estar disponíveis, porém não foram encontrados em virtude dos termos utilizados.

O levantamento foi realizado no mês de janeiro de 2013 e foram encontrados e lidos dezoito trabalhos. A amostra final do estudo foi composta por 07 artigos que atenderam aos critérios de seleção. Após a leitura de cada artigo, deu-se início á fase de análise dos mesmos. Para análise dos dados e síntese dos artigos foram utilizados os seguintes aspectos: ano, local de publicação, metodologia, local do estudo, população alvo, objetivos e resultados.

\section{RESULTADOS E DISCUSSÕES}

No presente estudo, optou-se por selecionar somente artigos produzidos no Brasil para conhecer a produção e preocupação com o tema no país. No que se refere à metodologia utilizada nos artigos avaliados 71,4\% (5) eram estudos qualitativos, 14,3\% (1) artigos de revisão e $14,3 \%$ (1) pesquisas quantitativas. A tabela 2, apresentada a seguir, mostra um panorama geral 
dos artigos analisados.

Em relação ao tipo de periódico, 42,8\% (3) foram publicados em periódicos de enfermagem, 28,6\% (2) artigos em um periódico de radiologia e 28,6\% (2) artigos em periódicos de ciências da saúde. Dentre os 7 artigos apresentados, 71,4\% (5) são de autoria de enfermeiros $^{(2,6,11,12,13)}$, sendo 42,8\% (3) deles de exclusivamente da categoria ${ }^{(6,11,12,13)}, 42,8 \%$ (3) são escritos por físicos ${ }^{(2,9,10)}, 42,8 \%$ (3) são de médicos ${ }^{(9,10,12)}$ e $14,3 \%$ (1) teve a colaboração de um farmacêutico ${ }^{(12)}$.

Em relação às variáveis estudadas nos artigos, emergiram questões relacionadas à condições de trabalho e procedimentos de biossegurança, ${ }^{(2,9,10,12)}$, atuação das enfermeiras ${ }^{(6,13)}$, legislação ${ }^{(2,9,10,12)}$, educação permanente $e^{(2,6,12)}$ e dificuldades e necessidades das equipes de enfermagem $^{(9,11)}$. Os resultados sistematizados são demonstrados na tabela 2. 
Tabela 2: Síntese dos achados

\begin{tabular}{|c|c|c|c|c|c|c|}
\hline Ano & $\begin{array}{c}\text { Local de } \\
\text { publicação }\end{array}$ & $\begin{array}{l}\text { Tipo de } \\
\text { pesquisa }\end{array}$ & $\begin{array}{c}\text { Local do } \\
\text { estudo }\end{array}$ & População alvo & Objetivos & Principais Resultados \\
\hline 2003 & $\begin{array}{c}\text { Revista } \\
\text { Radiologia } \\
\text { Brasileira }\end{array}$ & $\begin{array}{l}\text { Pesquisa } \\
\text { qualitativa }\end{array}$ & Rio de Janeiro & $\begin{array}{c}\text { Serviços e } \\
\text { funcionários } \\
\text { diretamente expostos } \\
\text { às radiações ionizantes }\end{array}$ & $\begin{array}{c}\text { Elaborar um Programa de } \\
\text { Monitoração Ocupacional } \\
\text { em Radiologia Médica } \\
\text { para o Hospital } \\
\text { Universitário. Clementino } \\
\text { Fraga Filho da UFRJ. }\end{array}$ & $\begin{array}{l}\text { Constatou-se que a maioria desses } \\
\text { funcionários não eram monitorados e } \\
\text { que não havia controle para os casos de } \\
\text { doses elevadas. }\end{array}$ \\
\hline 2005 & $\begin{array}{c}\text { Revista } \\
\text { Radiologia } \\
\text { Brasileira }\end{array}$ & $\begin{array}{l}\text { Pesquisa } \\
\text { qualitativa }\end{array}$ & $\begin{array}{c}\text { Hospital } \\
\text { público de } \\
\text { grande porte } \\
\text { da cidade do } \\
\text { RJ. }\end{array}$ & $\begin{array}{l}\text { Ténicos em radiologia } \\
\text { ou enfermeiras do } \\
\text { seviço de radiologia }\end{array}$ & $\begin{array}{c}\text { Avaliar as condições de } \\
\text { biossegurança e serviços } \\
\text { de radiologia para adequar } \\
\text { as normas vigentes do país. }\end{array}$ & $\begin{array}{c}\text { O sistema de ventilação era } \\
\text { insuficiente, os profissioanis não } \\
\text { utilizavam EPIs no preparo dos } \\
\text { produtos químicos. Aspectos } \\
\text { ergonômicos m desacordo com } \\
\text { normas nacioanis e os funcionários }\end{array}$ \\
\hline
\end{tabular}




\begin{tabular}{|c|c|c|c|c|c|c|}
\hline & & & & & & $\begin{array}{l}\text { apresentam estresse relacionado ao } \\
\text { trabalho }^{(10)} \text {. }\end{array}$ \\
\hline 2009 & $\begin{array}{c}\text { Revista } \\
\text { Arquivo de } \\
\text { Ciências da } \\
\text { Saúde }\end{array}$ & $\begin{array}{l}\text { Qualitativa, } \\
\text { exploratória e } \\
\text { descritiva. }\end{array}$ & $\begin{array}{c}30 \text { cidades } \\
\text { distribuídas } \\
\text { em } 17 \text { estados } \\
\text { do país }\end{array}$ & $\begin{array}{c}56 \text { enfermeiros } \\
\text { hemodinâmiccistas }\end{array}$ & $\begin{array}{l}\text { Identificar dificuldades e } \\
\text { necessidades da equipe de } \\
\text { enfermagem em serviços } \\
\text { de hemodinâmica e } \\
\text { angiografia no país. }\end{array}$ & $\begin{array}{c}\text { Os enfermeiros demostraram } \\
\text { dificuldade em gerenciar e liderar com } \\
\text { o setor de hemodinâmica, assim como } \\
\text { em executar diversas atividades. }\end{array}$ \\
\hline
\end{tabular}




\begin{tabular}{|c|c|c|c|c|c|c|}
\hline & & & & & & treinamento \\
\hline 2010 & $\begin{array}{l}\text { Jornal of the } \\
\text { Health } \\
\text { Sciences } \\
\text { Institute }\end{array}$ & $\begin{array}{c}\text { Estudo } \\
\text { qualitativo } \\
\text { descritivo }\end{array}$ & Goiânia-GO & $\begin{array}{l}\text { Enfermeiras atuantes } \\
\text { no Centro de } \\
\text { Diagnóstico por } \\
\text { imagem }\end{array}$ & $\begin{array}{l}\text { Conhecer a atuação das } \\
\text { enfermeiras que trabalham } \\
\text { no Centro de Diagnóstico } \\
\text { por Imagem de um hospital } \\
\text { público. }\end{array}$ & $\begin{array}{c}\text { A pesquisa resultou em } 2 \text { eixos } \\
\text { temáticos: conhecendo a realidade das } \\
\text { enfermiras no Centro de Diagnóstico } \\
\text { por Imagem-CDI e atuação das } \\
\text { enfermeiras no CDI. No primeiro eixo } \\
\text { constatou-se que não existe a consulta } \\
\text { de enfermagem; as enfermeiras não } \\
\text { participam de eventos, reuniões } \\
\text { cintíficas e discussões de casos } \\
\text { clínicos. O segundo eixo foi retratado } \\
\text { questões sobre a realidade das } \\
\text { enfermeiras, que compreendem as } \\
\text { funções: planejar, organizar, } \\
\text { supervisionar, executar e avaliar todas } \\
\text { as atividades de enfermagem em } \\
\text { clientes submetidas à radiação } \\
\text { ionizante. }\end{array}$ \\
\hline
\end{tabular}


2011 Revista Texto

\& Contexto

Enfermagem

Estudi

om desenho

transversal

8 técnicos em
radiologia

estado do RS

Região
noroeste do
estado do RS

S

\section{transversal}

\section{- 2015}


A análise dos artigos que compuseram a amostra desse estudo tornou possível a observação de que a grande preocupação dos pesquisadores em torno dessa área específica ocorre, principalmente, acerca das competências dos profissionais da enfermagem nos serviços de radiologia e diagnóstico por imagem, assim como dos conhecimentos demonstrados por cada um destes.

O COFEN ampara através da Resolução $n^{0}$ 211/98 ${ }^{(4)}$ a atuação do enfermeiro que trabalha nos serviços de radiodiagnóstico. Nesta regulamentação os profissionais são orientados sobre as medidas de proteção radiológicas e também sobre o treinamento dos profissionais para o uso correto dos equipamentos de proteção individual e radioproteção.

A radiologia compreende duas grandes áreas principais de atuação, para a enfermagem radiológica. O diagnóstico por imagem e a radiologia intervencionista/terapêutica ${ }^{(14)}$.

O processo de trabalho em um serviço de diagnóstico por imagem necessita de uma equipe multiprofissional, pois envolve diversos saberes, sendo impraticável a realização deste serviço por um profissional único. Cada profissional desenvolve sua assistência em sua área de conhecimento especifico, propiciando um resultado de maior e melhor qualidade. A equipe geralmente é composta de médico especialista em radiologia, radiofarmacêutico, físico, enfermeiro, técnico em radiologia e em enfermagem ${ }^{(6)}$.

Neste contexto observa-se que as principais competências da enfermagem nos serviços de diagnósticos por imagens estabelecem em realizar procedimentos técnicos, elaboração de questionários, orientações, protocolos, manuais, administração do setor, organização, treinamento dos profissionais de enfermagem, orientações e intervenções no preparo do paciente antes, durante e depois do exame ${ }^{(6,14)}$.

A radiologia é uma especialidade da área da saúde que vem sofrendo diversos avanços, deixando de ser considerada uma especialidade puramente de diagnóstico para a medicina invasiva, processual e curativa. Em resposta a esse avanço, o papel da equipe de enfermagem no serviço de radiologia passa por um processo de evolução, de maneira que, atualmente a enfermagem radiológica é um elemento integral no cuidado aos pacientes submetidos a procedimentos radiológicos nos períodos pré, intra e pós-procedimento radiológico ${ }^{(15)}$. 
Os profissionais da enfermagem que atuam em serviços de radiologia e diagnóstico por imagem devem conhecer os benefícios, mas também os riscos da exposição à radiação ionizante, e dessa maneira, se proteger da exposição desnecessária equipamentos de proteção específicos, ou mesmo, por meio do fator inverso do quadrado da distância $\left({ }^{6}\right)$.

Na execução é necessário o conhecimento de biossegurança, que consiste em um conjunto de ações com o objetivo de prevenir, diminuir ou eliminar os riscos que o profissional e o paciente possam estar expostos no momento da realização do exame, dentre eles o uso de EPI's (Equipamento de Proteção Individual), como: aventais de proteção, saias de proteção, protetores abdominais, luvas de proteção, mangas, protetor de gônadas para pacientes masculinos, entre outros e o uso de EPC’s (Equipamento de Proteção Coletiva), como: cabine de segurança, extintor de incêndio, etc ${ }^{(17)}$.

Haja vista que os avanços das tecnologias radiológicas revolucionaram as práticas em saúde, há a necessidade de uma educação permanente para a assistência de enfermagem, pois é através disso que ocorre a qualificação deste profissional. A orientação adequada aos profissionais da enfermagem deve vir com o intuito de capacitá-los para desempenhar um importante papel tanto na coordenação do serviço quanto no atendimento ao paciente e, principalmente, para sanar as dúvidas de toda a equipe de enfermagem envolvida com as práticas radiológicas realizadas no serviço ${ }^{(18)}$.

Para isso, aponta a necessidade de os profissionais da enfermagem que desejam atuar na área da radiologia e diagnóstico por imagem de passarem um tempo acompanhando os procedimentos lá realizados, assim como fazem nas outras áreas do conhecimento ${ }^{(15)}$.

\section{CONCLUSÃO}

Percebe-se que a enfermagem radiológica vem acompanhada de uma curva de aprendizagem íngreme para os profissionais que desejam ingressar nessa área, e que mesmo os enfermeiros mais experientes tem muito que aprender quando se trata de habilidades específicas 
do processo de trabalho nos serviços de radiologia e diagnóstico por imagem ${ }^{(15)}$.

Sendo a radiologia uma especialidade peculiar na área da saúde, para atuação da enfermagem, apresentando necessidades de conhecimentos gerais da sua formação e específicos de física e proteção radiológica, é extremamente importante apontar a necessidade de atualização desses profissionais, a fim de melhorar a qualidade da assistência ao paciente submetido a procedimentos radiológicos. Dessa maneira, a busca por um processo educativo contínuo deve ser constante, principalmente para os profissionais da enfermagem radiológica, os quais criam um elo com o paciente, visto que a sua permanência ao lado deste é maior do que qualquer outro profissional da saúde.

Além disso, a promoção de capacitações nos próprios serviços é um meio de fornecer subsídios teóricos aos profissionais, auxiliando no enriquecimento intelectual e de qualidade nos serviços prestados à população.

A atuação do enfermeiro com as tecnologias radiológicas deve estar embasada em conhecimento científico atualizado e com técnica apropriada, a fim de que se alcance o resultado clínico desejado e se promova a segurança e satisfação do paciente. 


\section{REFERÊNCIAS}

1. ALOTAIBI, M; SAEED; R. Radiology nurses' awareness of radiation. J. Radiology Nurs. 25(1)p. 7-12. mar. 2006.

2. BRAND I.C; FONTANA, R.T; SANTOS, Av. A saúde do trabalhador em radiologia: algumas considerações. Texto contexto - enferm, p 68-75. Jan-mar. 201.

3. BRUNI, K. R. The role of the vascular nurse in centers of excellence. J. Vascular Nurs. 20 (1) p 2-5. mar. 2002.

4. CNET, Center For Nursing Education And Testing Inc; RNCB, Radiologic Nursing Certification Board Inc. Practice analysis of radiology nursing. J. Radiology Nurs. 29(2): p. 109-28. Dez. 2010.

5. CONSELHO FEDERAL DE ENFERMAGEM. RESOLUÇÃO COFEN-211/1998: Dispõe sobre a atuação dos profissionais de Enfermagem que trabalham com radiação ionizante. Rio de Janeiro,1998.

6. CONSELHO FEDERAL DE ENFERMAGEM. RESOLUÇÃO COFEN-347/2009: Normatiza em âmbito Nacional a obrigatoriedade de haver Enfermeiro em todas as unidades de serviço onde são desenvolvidas ações de Enfermagem durante todo o período de funcionamento da instituição de saúde. Rio de Janeiro, 1998.

7. FERNANDES, G.S; CARVLHO, A. C. P; AZEVEDO, A. C. P. Avaliação dos riscos ocupacionais de trabalhadores de serviços de radiologia. Radiol. bras. 38(4): p. 279-81. Julago. 2005.

8. FERREIRA, Nsa. As pesquisas denominadas “estado da arte”. Educação \& Sociedade. p 257272. Ago. 2002

9. FLÔR, R C; LGELBCKE, F. Tecnologias emissoras de radiação ionizante e a necessidade de educação permanente para uma práxis segura da enfermagem radiológica.. Rev Bras Enferm, Brsilia,62(5) p.766-770, set-out. 2009.

10. GOODHART, J; PAGE, J. Orthopaedic Nursing. J. Radiologic Nurs. p 26 (1): 36-39. jan-fev. 2007.

11. KOCH, H. A; RIBEIRO, E. C. O; TONOMURA, E. T. Radiologia na Formação do Médico Geral. Rio de Janeiro: Revinter. 1997.

12. MINISTÉRIO DA SAÚDE. ORGANIZAÇÃO PAN-AMERICANA DA SAÚDE NO BRASIL: doenças relacionadas ao trabalho: manual de procedimentos para os serviços de saúde. 
Brasilia. 2001

13. Oliveira, S. R; AZEVEDO, A. C.P. Oliveira, S. R Elaboração de um programa de monitoração ocupacional em radiologia para o Hospital Universitário Clementino Fraga Filho. Radiol. bras. p . 36 (1). jan-fev. 2003.

14. PIRES, D. E. P. Reestruturação Produtiva e trabalho em Saúde no Brasil. 2. ed. São Paulo: Anna Blume, 2008.

15. SALES, O. P. OLIVEIRA C.C.C; SPIRANDELLI, F.A.P; CANDIDO, M.T. Atuação de enfermeiros em um Centro de Diagnóstico por Imagem. J. Health Sci. Inst. p . 28(4): 325-328. Out-dez. 2010.

16. SANTOS, B. J; HINRICHSEN, S. L; LIRA, C; VILLELA, T.A.S. Riscos ocupacionais em centros de radiodiagnóstico. Rev. enferm. UERJ. p . 18(3): 365-370. Set. 2010.

17. SOUSA, M.F. Management and leadership: educating and orienting the radiology nurse of the future. J. Radiology Nurs. p . 30(3):135-136. Set. 201.

18. VIEIRA, L.C; CONTRIN, L.M; ROL, J.L; CONTE, H.D; LIMA, A.R.S; CASTRO, E.D.R; SANTOS, M.L.T.. Dificuldades e necessidades da equipe de enfermagem em serviços de hemodinâmica e angiografia. Arq. ciênc. saúde. P . 16(1): 21-5. Jan-mar. 2009. 